\section{Birth weight and body fat mass in adults assessed by bioimpedance in the ELSA-Brasil study}

\author{
Peso ao nascer e adiposidade em adultos \\ avaliados com bioimpedância no estudo \\ ELSA-Brasil
}

\section{Peso al nacer y masa de grasa corporal en adultos evaluados por bioimpedancia en el estudio ELSA-Brasil}

\author{
Carolina Breda Resende 1 \\ Vivian Cristine Luft 1,2 \\ Bruce Duncan 1 \\ Rosane Harter Griep 3 \\ Maria del Carmen Bisi Molina 4 \\ Sandhi Maria Barreto 5 \\ Maria Ines Schmidt 1
}

doi: 10.1590/0102-311X00061619

\begin{abstract}
Intrauterine life is a critical period for the development of body fat and metabolic risk. This study investigated associations between birth weight and total and truncal body fat in adults. To do so, we analyzed data on 10,011 adults participating in the Brazilian Longitudinal Study of Adult Health (ELSA-Brasil) who self-reported birth weight as $<2.5 \mathrm{~kg}, 2.5-4.0 \mathrm{~kg}$, or $>4.0 \mathrm{~kg}$ at baseline (2008-2010) and underwent bioimpedance in the next follow-up visit (2012-2014). Greater mean total and truncal fat mass were seen in those with high birth weight compared with adequate birth weight $(p<0.001)$ in both sexes (total fat: $25.2 \mathrm{vs} .23 .1 \mathrm{~kg}$ in men and $31.4 \mathrm{vs} .27 .7 \mathrm{~kg}$ in women, and truncal fat: $13.5 \mathrm{vs} .12 .4 \mathrm{~kg}$ in men and $15.9 \mathrm{vs} .14 .2 \mathrm{~kg}$ in women). U-shaped patterns were observed in restricted cubic-spline analyses in the subset of 5,212 individuals reporting exact birth weights, although statistically significant only for those with high birth weight. In the whole sample, in comparing high to adequate birth weight, the latter predicted having a large ( $>85$ percentile) total and truncal fat mass, respectively: $O R=1.76,95 \%$ CI: 1.37-2.25 (men) and $O R=1.86,95 \%$ CI: 1.42-2.44 (women); OR $=1.68,95 \%$ CI: $1.31-2.16$ (men) and $\mathrm{OR}=1.73,95 \% \mathrm{CI}: 1.31-2.28$ (women). However, low birth weight predicted having a large ( $>85$ percentile) \% truncal fat only in women $(O R=1.40$, 95\% CI: 1.03-1.91). In conclusion, in these men and women born in a period in which fetal malnutrition was prevalent, birth weight showed complex, frequently non-linear associations with adult body fat, highlighting the need for interventions to prevent low and high birth weight during pregnancy.
\end{abstract}

Obesity; Birth Weight; Body Composition; Adipose Tissue;

Observational Study

\author{
Correspondence \\ V. C. Luft \\ Programa de Pós-graduação em Epidemiologia, Faculdade de \\ Medicina, Universidade Federal do Rio Grande do Sul. \\ Rua Ramiro Barcelos 2400, Porto Alegre, RS 90035-002, Brasil. \\ vcluft@hcpa.edu.br

\footnotetext{
1 Faculdade de Medicina, Universidade Federal do Rio Grande do Sul, Porto Alegre, Brasil.

2 Hospital das Clinicas de Porto Alegre, Porto Alegre, Brasil. 3 Instituto Oswaldo Cruz, Fundação Oswaldo Cruz, Rio de Janeiro, Brasil.

4 Centro de Ciências da Saúde, Universidade Federal do Espírito Santo, Vitória, Brasil.

5 Faculdade de Medicina, Universidade Federal de Minas
} \\ Gerais, Belo Horizonte, Brasil.
}




\section{Introduction}

Obesity, a major public health problem, is now the world's 6th most important risk factor for overall disease burden 1. It presents a high and growing prevalence in Brazil and the world 2, and causes hyperglycemia, hypertension, hypercholesterolemia, sleep apnea, and leads to type 2 diabetes, cardiovascular diseases, many cancers, chronic renal disease and a series of other conditions, including musculoskeletal ones, producing disease burden 1,3,4.

Obesity results from a complex multifactorial process, which involves not only lifestyle but also environmental exposures, social factors, socioeconomic conditions, family history and genetic factors 5 . Weight gain is manifested throughout the course of life, and obesity risk begins early in life, with the intrauterine period being critical in its development 6,7,8. Both low and high birth weight have been linked to greater risk of obesity in adulthood ${ }^{9}$, as well as to obesity-related metabolic problems such as diabetes and hypertension 10 . There is compelling evidence that intrauterine adversity not only restricts fetal growth, but also alters gene expression in a manner which favors increased future risk of adiposity and metabolic diseases 11,12 .

Body fat distribution, especially central obesity, has been shown to predict mortality better than overall adiposity 13 . Some studies have shown a relationship between birth weight and the distribution of body fat in adult life 14,15, but understanding on the development of different aspects of obesity within a life course perspective is still incipient. The distribution of body fat logically differs between men and women in response to the specific needs and stressors of males and females throughout evolution 16,17,18,19,20. Studies in human populations of low or high birth weight with body fat distribution in adult life found that responses to adverse exposures in early life are gender-specific 21,22,23,24,25,26,27,28,29. Previous analyses of the Brazilian Longitudinal Study of Adult Health (ELSA-Brasil) have shown gender-specific associations between birth weight and greater overall and central obesity, assessed by body mass index, waist circumference and waist-to-hip ratio 30 and additionally diabetes 31 in adult life. Almost all of the cited studies, however, have been based on anthropometric measurements. We are unaware of prior studies investigating how birth weight relates to adult adiposity obtained with bioimpedance.

Although anthropometric measurements may provide a useful indication of central obesity in a clinical setting, most studies have concluded the estimation of visceral fat using anthropometry is limited, accounting for between $50 \%$ and $80 \%$ of the variance in both sexes 32 . Emerging evidence points to the existence of many "obesity phenotypes", with differing associations to chronic diseases 33 . Bioimpedance analysis may contribute to a better understanding of adiposity distribution. In this context, this study aims to investigate the association of birth weight with total and truncal body fat, as evaluated by bioimpedance in adults.

\section{Method}

\section{Subjects}

This study reports a cross-sectional evaluation from the ELSA-Brasil, a cohort study whose main objective is to investigate factors related to the etiology of diabetes and cardiovascular diseases. Participants are active or retired civil servants from universities or research institutions, recruited between the ages of 35 and 74 years in capital cities of six Brazilian states: Rio de Janeiro, São Paulo, Rio Grande do Sul, Minas Gerais, Bahia, and Espírito Santo 34. Volunteers were invited through announcements and telephone calls, being the latter more commonly used to attract retirees. A randomly ordered list of employees, stratified by sex, age, and occupational category, was also used to actively recruit eligible participants. Efforts were made to recruit similar proportions of men and women, as well as predefined proportions of age groups and occupational categories 34 .

Of the initial sample of 15,105 participants at the cohort's baseline (2008-2010), 223 died between visits and 868 did not attend the follow-up visit (2012-2014). Additionally, 2,006 were excluded because of uncertain birth weight, 189 for twin birth, 650 for prematurity or uncertainty about pre- 
maturity, 741 for lack of valid bioimpedance data, 122 for previous bariatric surgery, and 295 for lack of information on covariates, leaving a total sample of 10,011 participants for analysis.

During the baseline exam, we asked participants to indicate their birth weight as "less than $2.5 \mathrm{~kg}$ " (low birth weight), "2.5-4.0kg" (normal birth weight), "greater than 4.0kg" (high birth weight) or "I do not know", in addition to their exact birth weight, if known.

At the follow-up visit, in the study center, body composition was evaluated by segmental bioimpedance with 8 electrodes (InBody 230; InBodyCo., Seoul, Korea), capable of distinguishing trunk and limb masses. Participants were instructed to fast for at least 8 hours, and not to ingest caffeine nor engage in physical activity within the 24 hours prior to examination. The participants urinated within minutes prior to measurement, wore standardized clothing and removed all metallic objects such as earrings, rings, glasses, etc. Bioimpedance was not performed on participants with pacemakers or metal prostheses.

Four outcome variables were defined based on fat mass as determined by bioimpedance: total body and truncal fat mass $(\mathrm{kg})$, and the relative proportions (\%) each comprised of total body weight.

Information concerning relevant covariates - preterm and twin birth, maternal and paternal history of diabetes, educational level, net family income per capita and age 34 - were obtained through interviews. Additionally, height, weight, waist and hip circumferences, and sagittal abdominal height were concomitantly measured. The staff obtaining these data were previously trained and certified, following ELSA-Brasil's quality assurance and control routines 35 .

\section{Statistical analysis}

We describe the characteristics of participants as absolute and relative frequencies for categorical variables, and mean (standard deviation) or median (25-75 percentile) for continuous ones. Scatter plots and Spearman correlation coefficients were used to describe the relationship between body composition when estimated by bioimpedance and anthropometry (body mass index - BMI, waist circumference, hip circumference, waist-hip ratio, waist/height, sagittal abdominal height).

The distribution of covariates across birth weight categories, testing crude differences with the chi-square test for categorical variables and analysis of variance for continuous ones, is presented separately for men and women. Means of total fat mass $(\mathrm{kg})$, total fat mass/weight (\% body fat), trunk fat mass $(\mathrm{kg})$ and trunk fat mass/weight (\% truncal fat), are presented by sex, according to birth weight and to categories of age, net family income per capita, race/color, educational level, mother's educational level, and maternal and paternal history of diabetes, adjusted in analysis of variance.

High values of body composition were defined as those above the 85th percentile of the distribution of each body composition variable and estimated the odds of those reporting low or high birth weight, compared to appropriate birth weight, to present these high values, adjusting through logistic regression for covariates.

In futher analyses, in participants who informed their specific birth weight $(\mathrm{n}=5,212)$, we employed restricted cubic spline multiple linear regression, with nodes defined at percentiles 10, 50 and 9036 to estimate the differences in total or truncal body fat, as a percentage of total weight, across the continuously expressed spectrum of birth weight, in comparison to values for a $3.2 \mathrm{~kg}$ birth weight. A similar approach was used, but with logistic regression, to estimate the adjusted odds of having a high percentage ( $>85$ th percentile) of total or truncal fat across the continuously expressed spectrum of birth weight, also when compared to values for a $3.2 \mathrm{~kg}$ birth weight. All analyses were performed in SAS, version 9.4 (https://www.sas.com), and statistical significance was defined as $\mathrm{p}<0.05$. 


\section{Results}

As can be seen in Table 1, 5,490 (55\%) participants were women, and 5,510 (55\%) declared themselves white, 2,687 (27\%) brown, 1,501 (15\%) black, 224 (2\%) yellow (Asian) and 89 (1\%) indigenous. Low birth weight $(<2.5 \mathrm{~kg})$ was reported by $529(5 \%)$ and high birth weight (> 4.0kg) by $764(8 \%)$. Median monthly family income per capita was USD 984.79 (25 percentile-75 percentile: $604.65-1,641.32$ ), and $61 \%$ of participants had completed college. The majority $(54.5 \%)$ of mothers of participants, in terms of schooling, had not completed elementary school. A maternal history of diabetes was reported by 1,970 (20\%) and a paternal history of diabetes by 1,329 (13\%). Mean age was $55.0 \pm 8.7$ years when bioimpedance analysis was performed. This sample is quite similar to the overall sample of ELSA-Brasil (where 54.4\% were women, 52\% declared themselves white, 28\% brown, 16\% black, in second visit mean age was $55.7 \pm 9.0$, median monthly family income per capita was USD 950.21 (25 percentile-75 percentile: $583.08-1,468.54), 57 \%$ of participants had completed college, $57 \%$ of mothers of participants had not completed elementary school, low birth weight was reported by $8.4 \%$ and high birth weight by $7.5 \%$. Among men, mean BMI was $27.4 \pm 4.3 \mathrm{~kg} / \mathrm{m}^{2}$, waist circumference $95.2 \pm 11.6 \mathrm{~cm}$, hip circumference $100.4 \pm 8.0 \mathrm{~cm}$, and abdominal height $22.2 \pm 3.4 \mathrm{~cm}$. Among women, mean BMI was $27.7 \pm 5.2 \mathrm{~kg} / \mathrm{m}^{2}$, waist $87.3 \pm 12.2 \mathrm{~cm}$, hip $103.0 \pm 10.3 \mathrm{~cm}$, and abdominal height $20.7 \pm 4.1 \mathrm{~cm}$.

Table 1 also presents frequency or mean values of these characteristics by birth weight, separately for men and women. Individuals reporting low birth weight had lower educational attainment and household income per capita. The distribution of maternal and paternal history of diabetes across categories of birth weight was not consistent among men and women. A maternal history of diabetes was greater among those with high birth weight.

Figure 1 shows the correlations of our four bioimpedance outcome variables with traditional anthropometric measurements. Absolute measures of bioimpedance (total and truncal fat) correlated strongly with BMI ( $r \geq 0.91)$, abdominal height $(r \geq 0.88)$, and waist circumference $(r \geq 0.82)$, with high correlations both in men and women. Relative measures of bioimpedance (total fat/weight, truncal fat/weight) were less correlated, being stronger with BMI $(r \geq 0.77)$, abdominal height $(r \geq 0.76)$ and waist/height $(r \geq 0.72)$.

Table 2 describes adjusted means of total body and truncal fat assessed by bioimpedance, according to birth weight and sociodemographic characteristics, separately for men and women. Women had higher values of body fat, both in absolute terms and concerning body weight. Absolute measurements of body fat mass (total and truncal) increased with birth weight ( $<<0.001)$, in both sexes. Fat and truncal fat as percentages of body weight had slightly higher values in the low and high birth weight categories for both men and women, though without statistical significance in comparison with those of appropriate birth weight $(\mathrm{p}>0.85)$. There was no difference regarding sex in these associations ( $\mathrm{p} \geq 0.87$ for the interaction between birth weight and sex).

As also seen in Table 2, older men and women presented greater amounts of both overall and truncal fat, both in relative and absolute terms. However, women who declared being of white/race color had lower mean values of fat indices, while men had higher values. Similar differences by sex were also seen across income and educational attainment; women with higher income and educational attainment having higher values, while men, lower ones. Individuals with a maternal or paternal history of diabetes had uniformly greater indices.

Restricted cubic spline analyses for the 5,212 participants who reported exact birth weights (Figure 2) show a general U-shaped pattern of mean differences of relative total body and truncal fat across birth weight categories. In these analyses, the weight nadir was always at or near a birth weight of $3.2 \mathrm{~kg}$. However, only the greater mean percentage fat values seen with high birth weight achieved statistical significance, as indicated by the low border of the shaded zones of $95 \%$ confidence being above the line of zero difference.

To examine the magnitude of the associations of birth weight categories with high ( $>85$ percentile) values of absolute and relative total and truncal fat, the next logistic regression analyses perfomed were adjusted for study center, gender, age, family income, race/color, participant's and mother's educational attainment. As seen in Figure 3 (to the left, for absolute amount of body fat), individuals of both sexes with high (vs. appropriate) birth weight had greater odds of having high total fat mass (men: $\mathrm{OR}=1.76,95 \% \mathrm{CI}: 1.37-2.25$; women: $\mathrm{OR}=1.86,95 \% \mathrm{CI}: 1.42-2.44$ ), as well as high truncal fat 
Table 1

Characteristics of Brazilian Longitudinal Study of Adult Health (ELSA-Brasil) participants according to birth weight and sex.

\begin{tabular}{|c|c|c|c|c|c|c|c|c|c|}
\hline & \multirow{2}{*}{$\begin{array}{c}\text { Total } \\
(\mathrm{N}=10,011)\end{array}$} & \multicolumn{4}{|c|}{$\operatorname{Men}(n=4,521)$} & \multicolumn{4}{|c|}{ Women $(n=5,490)$} \\
\hline & & $\begin{array}{c}<2.5 \mathrm{~kg} \\
(n=234)\end{array}$ & $\begin{array}{c}2.5-4.0 \mathrm{~kg} \\
(\mathrm{n}=3,850)\end{array}$ & $\begin{array}{c}>4.0 \mathrm{~kg} \\
(n=437)\end{array}$ & p-value & $\begin{array}{c}<2.5 \mathrm{~kg} \\
(\mathrm{n}=294)\end{array}$ & $\begin{array}{c}2.5-4.0 \mathrm{~kg} \\
(\mathrm{n}=4,869)\end{array}$ & $\begin{array}{c}>4.0 \mathrm{~kg} \\
(n=327)\end{array}$ & p-value \\
\hline Age & $55.0 \pm 8.8$ & $55.9 \pm 9.0$ & $55.0 \pm 9.0$ & $53.8 \pm 8.8$ & 0.01 * & $55.6 \pm 8.6$ & $55.0 \pm 8.6$ & $54.6 \pm 8.1$ & 0.35 * \\
\hline Race/Color & & & & & $<0.01 * *$ & & & & $0.03 * *$ \\
\hline White & $5,510(55.0)$ & $101(43.0)$ & $2,145(55.7)$ & $270(61.8)$ & & $138(46.9)$ & $2,659(54.6)$ & $197(60.2)$ & \\
\hline Brown & 2,687 (26.8) & 81 (34.6) & $1,116(29.0)$ & $105(24.0)$ & & $92(31.3)$ & $1,221(25.1)$ & $72(22.0)$ & \\
\hline Black & 1,501 (15.0) & $40(17.0)$ & $488(12.7)$ & $51(11.7)$ & & $53(18.0)$ & $818(16.8)$ & $51(15.6)$ & \\
\hline Yellow (Asian) & $224(2.2)$ & $8(3.4)$ & $67(1.7)$ & $2(0.5)$ & & $11(3.7)$ & $131(2.7)$ & $5(1.5)$ & \\
\hline Indigenous & $89(0.9)$ & $4(1.7)$ & $34(0.9)$ & $9(2.1)$ & & $0(0.0)$ & $40(0.8)$ & $2(0.6)$ & \\
\hline $\begin{array}{l}\text { Per capita family } \\
\text { income }\end{array}$ & & & & & $<0.01$ * & & & & $<0.01$ * \\
\hline $\begin{array}{l}2014 \text { minimum } \\
\text { wages } * \star \star\end{array}$ & $3.3(2.0-5.4)$ & $2.1(1.4-3.9)$ & $3.2(1.9-4.9)$ & $3.3(2.0-5.4)$ & & $3.7(2.0-5.4)$ & $3.7(2.1-5.4)$ & $3.7(2.1-5.6)$ & \\
\hline $\begin{array}{l}\text { Educational } \\
\text { attainment }\end{array}$ & & & & & $<0.01$ ** & & & & $<0.01 * *$ \\
\hline $\begin{array}{l}\text { Incomplete } \\
\text { elementary }\end{array}$ & $363(3.6)$ & $27(11.5)$ & $196(5.1)$ & $19(4.4)$ & & $16(5.4)$ & $99(2.0)$ & $6(1.8)$ & \\
\hline Elementary & $527(5.3)$ & $29(12.4)$ & $256(6.7)$ & $18(4.1)$ & & $19(6.5)$ & $197(4.1)$ & $8(2.5)$ & \\
\hline High school & $3,020(30.2)$ & $89(38.0)$ & $1,177(30.6)$ & $113(25.9)$ & & 99 (33.7) & $1,443(29.6)$ & $99(30.3)$ & \\
\hline College & 6,101 (60.9) & $89(38.0)$ & $2,221(57.7)$ & 287 (65.7) & & $160(54.4)$ & $3,130(64.3)$ & $214(65.4)$ & \\
\hline $\begin{array}{l}\text { Mother's } \\
\text { educational } \\
\text { attainment }\end{array}$ & & & & & $<0.01$ ** & & & & $0.09 * *$ \\
\hline $\begin{array}{l}\text { Incomplete } \\
\text { elementary }\end{array}$ & $5,454(54.5)$ & $158(67.5)$ & $2,059(53.5)$ & 204 (46.7) & & $187(63.6)$ & $2,672(54.9)$ & $174(53.2)$ & \\
\hline Elementary & $2,023(20.2)$ & $34(14.5)$ & 769 (20.0) & 102 (23.3) & & 49 (16.7) & $1,004(20.6)$ & 65 (19.9) & \\
\hline High school & 1,826 (18.2) & $35(15.0)$ & 743 (19.3) & $91(20.8)$ & & 45 (15.3) & $847(17.4)$ & 65 (19.9) & \\
\hline College & $708(7.1)$ & $7(3.0)$ & $279(7.3)$ & $40(9.2)$ & & $13(4.4)$ & $346(7.1)$ & $23(7.0)$ & \\
\hline Maternal diabetes & & & & & $0.12 * \star$ & & & & $<0.01 * *$ \\
\hline Yes & 1,969 (19.7) & $45(19.2)$ & $733(19.0)$ & $101(23.0)$ & & $58(19.7)$ & $934(19.2)$ & $98(30.0)$ & \\
\hline No & $8,042(80.3)$ & $189(80.8)$ & $3,117(81.0)$ & 336 (76.9) & & $236(80.3)$ & $3,935(80.8)$ & $229(70.0)$ & \\
\hline Paternal diabetes & & & & & $0.01 * *$ & & & & $0.76 * *$ \\
\hline Yes & 1,329 (13.3) & $14(6.0)$ & $482(12.5)$ & $53(12.1)$ & & $41(14.0)$ & $688(14.1)$ & $51(15.6)$ & \\
\hline No & 8,682 (86.8) & $220(94.0)$ & $3,368(87.5)$ & 384 (87.9) & & $235(86.1)$ & $4,181(85.9)$ & $276(84.4)$ & \\
\hline
\end{tabular}

Note: age values expressed as mean (standard deviation), per capita family income values expressed as median (25 percentile-75 percentile), and all other variables values expressed as $n(\%)$.

* Analysis of variance;

** Chi-square test;

*** Minimum wage (per month) in $2014=$ BRL 724.00.

mass (men: $\mathrm{OR}=1.68,95 \% \mathrm{CI}: 1.31-2.16$; women: $\mathrm{OR}=1.73,95 \% \mathrm{CI}: 1.31-2.28$ ). However, for those with low birth weight, statistically significant (or near significant) lower odds of high total (OR $=0.65$, 95\%CI: $0.42-1.00)$ and truncal fat $(\mathrm{OR}=0.62,95 \% \mathrm{CI}$ : 0.40-0.96) mass were seen for men, but not for women $(\mathrm{OR}=0.96,95 \% \mathrm{CI}: 0.69-1.35$ and $\mathrm{OR}=0.99,95 \% \mathrm{CI}$ : 0.71-1.39).

As also seen in Figure 3 (to the right, for relative amount of fat), although sex interactions were not statistically significant ( $\mathrm{p}>0.11$ ), women presented U-shaped associations between birth weight and fat as a percentage of body weight, while men showed no association. As seen in the bottom figure, women with low birth weight showed higher odds of presenting a high truncal fat percentage 
Figure 1

Correlation between absolute $(\mathrm{kg})$ and relative (fat/weight) body composition, as estimated by bioimpedance and anthropometrics. Brazilian Longitudinal Study of Adult Health (ELSA-Brasil).

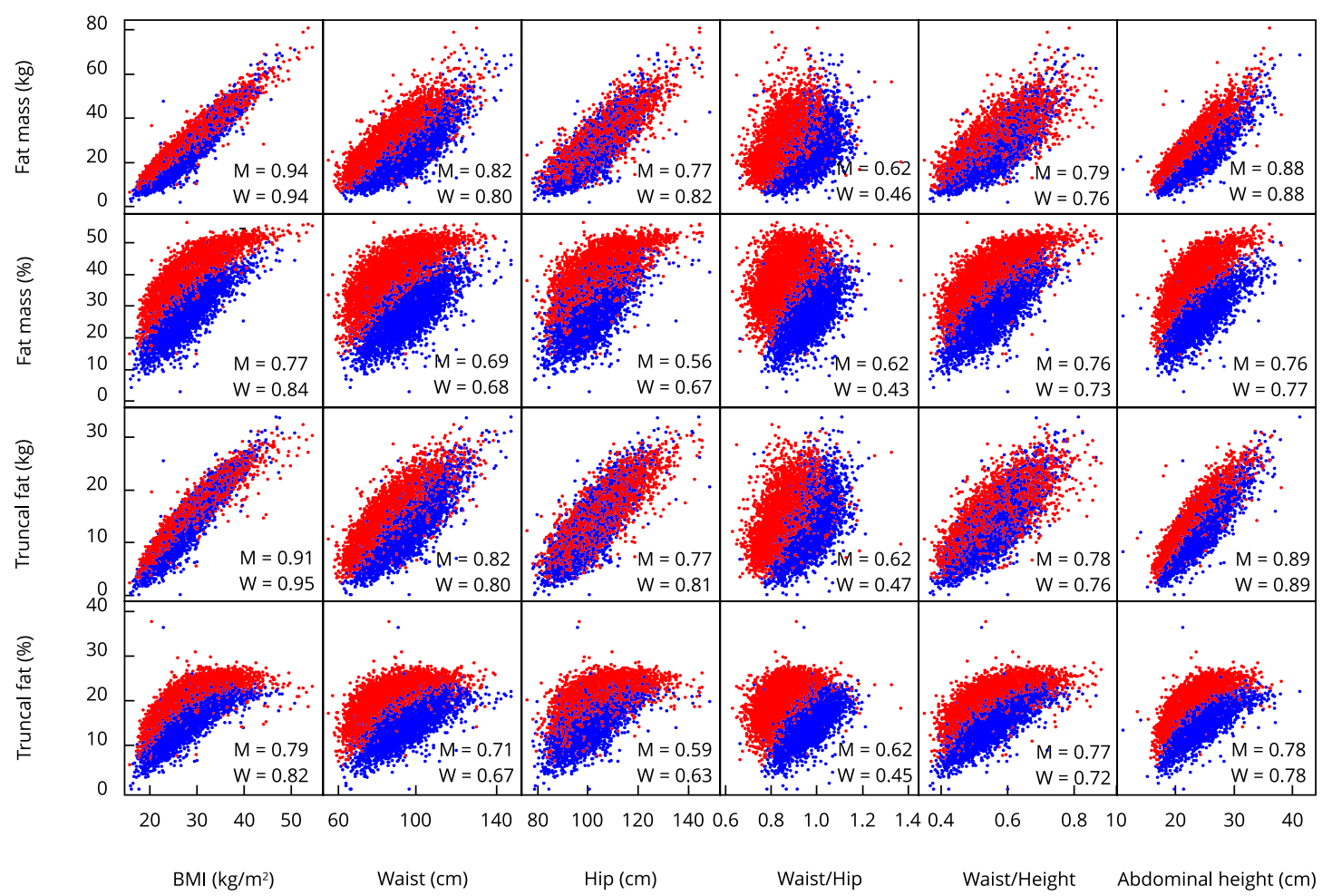

BMI: body mass index.

Note: blue = men $(M)$; red $=$ women $(\mathrm{W})$.

$(\mathrm{OR}=1.40$, 95\%CI: 1.03-1.91), while men $\operatorname{did}$ not $(\mathrm{OR}=1.04,95 \% \mathrm{CI}$ : 0.72-1.49). A similar pattern of associations with low birth weight was seen when examining high total body fat percentage (top figure). Similarly, though not statistically significant, women with high birth weight had greater relative odds of both truncal (OR = 1.11, 95\%CI: 0.81-1.54) and total body fat (OR = 1.34, 95\%CI: 0.99-1.80) as a percentage of weight, while men $\operatorname{did} \operatorname{not}(\mathrm{OR}=0.91,95 \% \mathrm{CI}$ : $0.67-1.23$ and $(\mathrm{OR}=0.99,95 \% \mathrm{CI}$ : $0.74-1.33)$

\section{Discussion}

Our measurements of body fat, assessed by bioimpedance in middle-age and elderly subjects, correlated strongly with measurements of BMI, sagittal diameter and waist-to-height ratios. When relating absolute measures of body fat with birth weight, we found generally positive associations. However, when comparing fat as a percentage of body weight, men and women both presented non-linear distributions. Notably, both men and women at birth weight extremes presented proportionally greater fat content, both overall and truncal, as adults, with these associations for high birth weight being statistically significant. However, in parallel analyses considering the relative odds of presenting a high portion of body weight as fat, only women maintained this U-shaped association, showing statistically greater risk of presenting high truncal fat if born with low birth weight, and a trend toward greater 
Table 2

Adjusted bioimpedance measures of body fat according to birth weight and socio-demographic characteristics, separately for men and women. Brazilian Longitudinal Study of Adult Health (ELSA-Brasil) $(N=10,011)$. Brazilian Longitudinal Study of Adult Health (ELSA-Brasil) $(N=10,011)$.

\begin{tabular}{|c|c|c|c|c|c|c|c|c|}
\hline & \multicolumn{2}{|c|}{ Total fat (kg) } & \multicolumn{2}{|c|}{ Total fat (\%) } & \multicolumn{2}{|c|}{ Truncal fat (kg) } & \multicolumn{2}{|c|}{ Truncal fat (\%) } \\
\hline & $\begin{array}{c}\text { Men } \\
\text { Mean }(95 \% \mathrm{Cl})\end{array}$ & $\begin{array}{c}\text { Women } \\
\text { Mean }(95 \% \mathrm{Cl})\end{array}$ & $\begin{array}{c}\text { Men } \\
\text { Mean }(95 \% \mathrm{Cl})\end{array}$ & $\begin{array}{c}\text { Women } \\
\text { Mean }(95 \% \mathrm{Cl})\end{array}$ & $\begin{array}{c}\text { Men } \\
\text { Mean }(95 \% \mathrm{Cl})\end{array}$ & $\begin{array}{c}\text { Women } \\
\text { Mean }(95 \% \mathrm{Cl})\end{array}$ & $\begin{array}{c}\text { Men } \\
\text { Mean }(95 \% \mathrm{Cl})\end{array}$ & $\begin{array}{c}\text { Women } \\
\text { Mean }(95 \% \mathrm{Cl})\end{array}$ \\
\hline \multicolumn{9}{|l|}{ Birth weight (kg) } \\
\hline$<2.5$ & $\begin{array}{c}21.8 \\
(21.0-23.0)\end{array}$ & $\begin{array}{c}27.1 \\
(26.0-28.3)\end{array}$ & $\begin{array}{c}27.9 \\
(27.0-28.8)\end{array}$ & $\begin{array}{c}39.0 \\
(38.2-39.5)\end{array}$ & $\begin{array}{c}11.6 \\
(11.0-12.3)\end{array}$ & $\begin{array}{c}13.9 \\
(13.3-14.5)\end{array}$ & $\begin{array}{c}14.9 \\
(14.5-15.4)\end{array}$ & $\begin{array}{c}20.1 \\
(19.7-20.6)\end{array}$ \\
\hline $2.5-4.0$ & $\begin{array}{c}23.1 \text { * } \\
(22.6-23.5)\end{array}$ & $\begin{array}{c}27.7 \text { * } \\
(27.6-28.2)\end{array}$ & $\begin{array}{c}27.6 \text { * } \\
(27.3-27.9)\end{array}$ & $\begin{array}{c}38.6 \text { * } \\
(38.3-39.0)\end{array}$ & $\begin{array}{c}12.4 \text { * } \\
(12.1-12.6)\end{array}$ & $\begin{array}{c}14.2 \text { * } \\
(13.9-14.4)\end{array}$ & $\begin{array}{c}14.8 \text { * } \\
(14.6-15.0)\end{array}$ & $\begin{array}{c}19.9 * \\
(19.7-20.1)\end{array}$ \\
\hline$>4.0$ & $\begin{array}{c}25.2 \text { ** } \\
(24.3-26.2)\end{array}$ & $\begin{array}{c}31.4 \text { ** } \\
(30.3-32.5)\end{array}$ & $\begin{array}{c}28.1 \\
(27.4-28.8)\end{array}$ & $\begin{array}{c}39.4 \\
(38.6-38.9)\end{array}$ & $\begin{array}{c}13.5 * * \\
(13.1-14.0)\end{array}$ & $\begin{array}{c}15.9 * \star \\
(15.3-16.5)\end{array}$ & $\begin{array}{c}15.2 \\
(14.8-15.5)\end{array}$ & $\begin{array}{c}20.1 \\
(19.7-20.6)\end{array}$ \\
\hline \multicolumn{9}{|l|}{ Age (years) } \\
\hline$<60$ & $\begin{array}{c}23.0 * \\
(22.5-23.5)\end{array}$ & $\begin{array}{c}27.9 * \\
(27.4-28.4)\end{array}$ & $\begin{array}{c}27.2 \text { * } \\
(26.8-27.6)\end{array}$ & $\begin{array}{c}38.4 \text { * } \\
(38.1-39.5)\end{array}$ & $\begin{array}{c}12.4 \text { * } \\
(12.1-12.6)\end{array}$ & $\begin{array}{c}14.3 \text { * } \\
(14.0-14.5)\end{array}$ & $\begin{array}{c}14.6 \text { * } \\
(14.5-14.8)\end{array}$ & $\begin{array}{c}19.8 \text { * } \\
(19.6-20.0)\end{array}$ \\
\hline$>60$ & $\begin{array}{c}24.0 * * \\
(23.4-24.6)\end{array}$ & $\begin{array}{c}28.5 \\
(27.7-29.0)\end{array}$ & $\begin{array}{c}29.5 * * \\
(29.0-30.0)\end{array}$ & $\begin{array}{c}40.1 \text { ** } \\
(39.6-40.5)\end{array}$ & $\begin{array}{c}12.8 \\
(12.5-13.1)\end{array}$ & $\begin{array}{c}14.5 \\
(14.2-14.8)\end{array}$ & $\begin{array}{c}15.8 * * \\
(15.5-16.0)\end{array}$ & $\begin{array}{c}20.1 * * \\
(20.4-20.8)\end{array}$ \\
\hline \multicolumn{9}{|l|}{ Race/Color } \\
\hline White & $\begin{array}{c}23.6 \text { * } \\
(23.0-24.1)\end{array}$ & $\begin{array}{c}27.3 \text { * } \\
(26.8-27.9)\end{array}$ & $\begin{array}{c}28.0 * \\
(27.6-28.4)\end{array}$ & $\begin{array}{c}38.5 \text { * } \\
(38.1-38.9)\end{array}$ & $\begin{array}{c}12.6 \text { * } \\
(12.4-12.9)\end{array}$ & $\begin{array}{c}14.0 \text { * } \\
(13.7-14.2)\end{array}$ & $\begin{array}{c}15.1 \text { * } \\
(14.9-15.3)\end{array}$ & $\begin{array}{c}19.8 \text { * } \\
(19.6-20.0)\end{array}$ \\
\hline Non-white & $\begin{array}{c}22.7 \text { ** } \\
(22.2-23.3)\end{array}$ & $\begin{array}{c}28.6 * * \\
(28.1-29.2)\end{array}$ & $\begin{array}{c}27.3 * * \\
(26.9-27.7)\end{array}$ & $\begin{array}{c}39.0 \\
(38.6-39.4)\end{array}$ & $\begin{array}{c}12.2 * * \\
(11.9-12.4)\end{array}$ & $\begin{array}{c}14.6 * * \\
(14.4-14.9)\end{array}$ & $\begin{array}{c}14.6 \text { ** } \\
(14.4-14.8)\end{array}$ & $\begin{array}{c}20.1 * * \\
(19.9-20.3)\end{array}$ \\
\hline \multicolumn{9}{|l|}{$\begin{array}{l}\text { Per capita family } \\
\text { income }\end{array}$} \\
\hline$\leq$ Median & $\begin{array}{c}22.7 * \\
(22.2-23.2)\end{array}$ & $\begin{array}{c}29.0 * \\
(28.5-29.6)\end{array}$ & $\begin{array}{c}27.5 * \\
(27.1-27.8)\end{array}$ & $\begin{array}{c}39.4 \text { * } \\
(39.0-39.8)\end{array}$ & $\begin{array}{c}12.2 \text { * } \\
(12.0-12.5)\end{array}$ & $\begin{array}{c}14.8 \text { * } \\
(14.5-15.1)\end{array}$ & $\begin{array}{c}14.8 \text { * } \\
(14.6-15.0)\end{array}$ & $\begin{array}{c}20.3 \text { * } \\
(20.0-20.5)\end{array}$ \\
\hline > Median & $\begin{array}{c}24.1 * \star \\
(23.5-24.7)\end{array}$ & $\begin{array}{c}27.2 \text { ** } \\
(26.7-27.8)\end{array}$ & $\begin{array}{c}28.2 \text { ** } \\
(27.8-28.6)\end{array}$ & $\begin{array}{c}38.4 \text { ** } \\
(38.0-38.8)\end{array}$ & $\begin{array}{c}12.9 * * \\
(12.6-13.2)\end{array}$ & $\begin{array}{c}13.9 * \star \\
(13.6-14.2)\end{array}$ & $\begin{array}{c}15.1 * \star \\
(14.9-15.4)\end{array}$ & $\begin{array}{c}19.7 \text { ** } \\
(19.5-19.3)\end{array}$ \\
\hline \multicolumn{9}{|l|}{$\begin{array}{l}\text { Educational } \\
\text { attainment }\end{array}$} \\
\hline College & $\begin{array}{c}23.6 \text { * } \\
(23.2-24.0)\end{array}$ & $\begin{array}{c}27.0 * \\
(26.7-27.4)\end{array}$ & $\begin{array}{c}27.7 \text { * } \\
(27.4-27.9)\end{array}$ & $\begin{array}{c}38.1 \text { * } \\
(37.8-38.3)\end{array}$ & $\begin{array}{c}12.6 \text { * } \\
(12.4-12.8)\end{array}$ & $\begin{array}{c}13.8 \text { * } \\
(13.6-14.0)\end{array}$ & $\begin{array}{c}14.9 * \\
(14.7-15.0)\end{array}$ & $\begin{array}{c}19.5 \text { * } \\
(19.4-19.7 .1)\end{array}$ \\
\hline$<$ College & $\begin{array}{c}22.5 * \star \\
(22.0-23.0)\end{array}$ & $\begin{array}{c}29.2 * \star \\
(28.7-29.7)\end{array}$ & $\begin{array}{c}27.3 \\
(27.0-27.7)\end{array}$ & $\begin{array}{c}39.4 * * \\
(39.0-39.8)\end{array}$ & $\begin{array}{c}12.1 * * \\
(11.9-12.4)\end{array}$ & $\begin{array}{c}15.0 * * \\
(14.7-15.2)\end{array}$ & $\begin{array}{c}14.7 \\
(14.5-14.9)\end{array}$ & $\begin{array}{c}20.3 \text { ** } \\
(20.1-20.5)\end{array}$ \\
\hline \multicolumn{9}{|l|}{$\begin{array}{l}\text { Mother's } \\
\text { educational } \\
\text { attainment }\end{array}$} \\
\hline$\geq$ High school & $\begin{array}{c}24.1 * \\
(23.3-24.6)\end{array}$ & $\begin{array}{c}27.2 * \\
(26.6-27.9)\end{array}$ & $\begin{array}{c}27.9 * \\
(27.4-28.4)\end{array}$ & $\begin{array}{c}38.2 \text { * } \\
(37.8-38.7)\end{array}$ & $\begin{array}{c}12.8 \text { * } \\
(12.5-13.2)\end{array}$ & $\begin{array}{c}13.9 * \\
(13.6-14.2)\end{array}$ & $\begin{array}{c}15.4 \text { * } \\
(15.1-15.6)\end{array}$ & $\begin{array}{c}20.0 \text { * } \\
(19.7-20.2)\end{array}$ \\
\hline$<$ High school & $\begin{array}{c}22.9 * * \\
(22.4-23.3)\end{array}$ & $\begin{array}{c}28.1 \text { ** } \\
(27.6-28.5)\end{array}$ & $\begin{array}{c}27.8 \\
(27.5-28.1)\end{array}$ & $\begin{array}{c}39.1 \text { ** } \\
(38.8-39.4)\end{array}$ & $\begin{array}{c}12.3 * * \\
(12.1-12.5)\end{array}$ & $\begin{array}{c}14.4 \text { ** } \\
(14.2-14.6)\end{array}$ & $\begin{array}{c}15.0 \\
(14.8-15.3)\end{array}$ & $\begin{array}{c}19.6 \text { ** } \\
(19.4-19.9)\end{array}$ \\
\hline
\end{tabular}

95\% Cl: 95\% confidence interval.

Note: values are adjusted through variance analysis, for study center, gender, and the other variables in this table.

* Reference for comparison;

$* *$ p-value $<0.05$

risk with high birth weight. To our knowledge, non-linear associations of birth weight with measurements of body composition as determined by bioimpedance have not been previously described.

Accumulating evidence regarding the associations of birth weight with overall/central obesity have produced heterogenous results across studies and populations. A systematic review documented a positive association with BMI but an inverse association with truncal obesity assessed by the ratio of 
Figure 2

Estimated differences in bioimpedance measures of \% body and truncal fat (fat/weight; reference: $3.2 \mathrm{~kg}$ birth weight), in restricted cubic spline analyses adjusted for study center, sex, age, income, race/color, participant's and mother's educational attainment. Brazilian Longitudinal Study of Adult Health (ELSA-Brasil) $(\mathrm{N}=10,011)$.

2a) All (body fat)

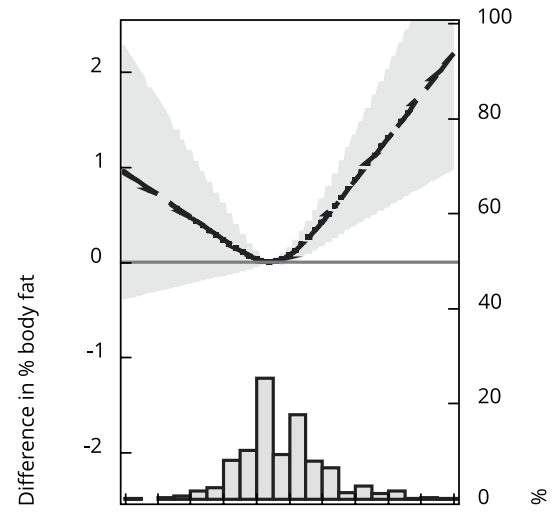

Birth weight (kg)

2d) All (truncal fat)

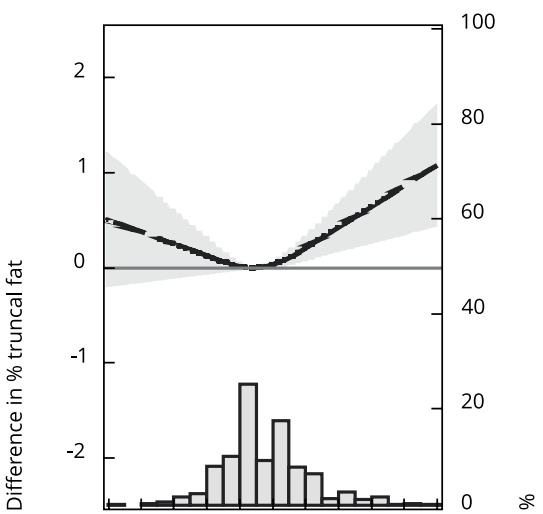

Birth weight $(\mathrm{kg})$ 2b) Women (body fat)

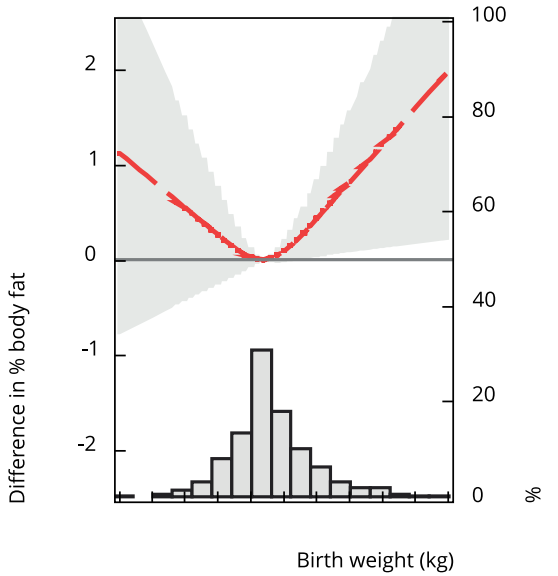

2e) Women (truncal fat)

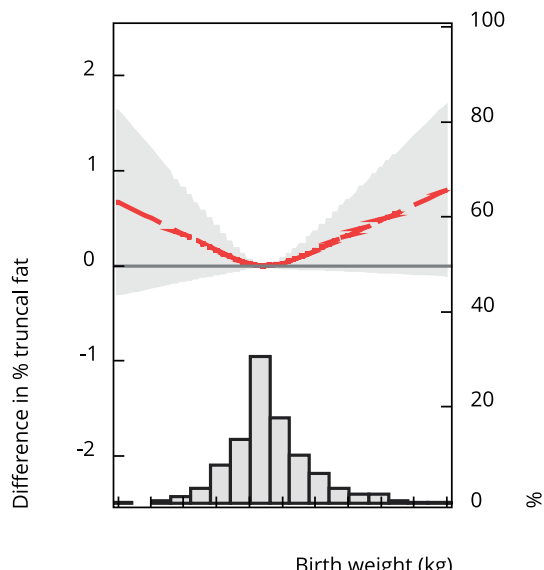

2c) Men (body fat)

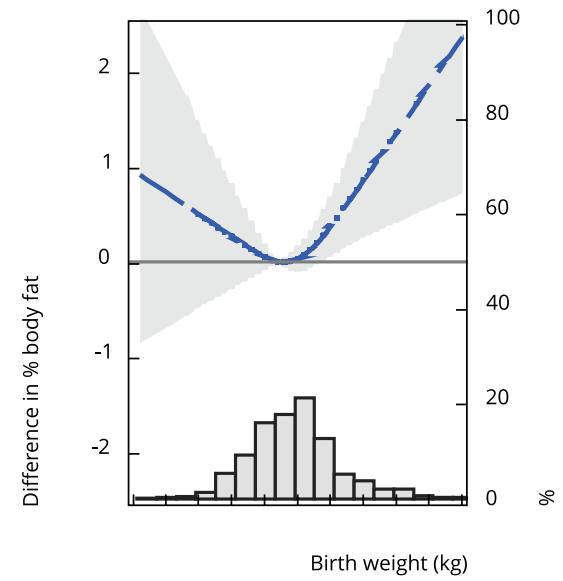

2f) Men (truncal fat)

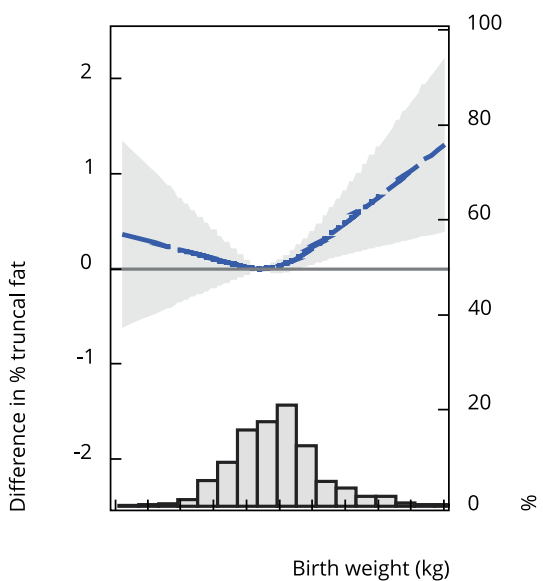

Note: black = all $(n=5,212)$; red = women $(n=2,804)$; blue = men $(n=2,408)$. Distributions of birth weight are superimposed.

subscapular/triceps skinfolds 14 . Positive associations with adult obesity assessed by body mass index were also documented in a systematic review 9 . The relationship of birth weight with central fatness in adults was further summarized by another systematic review, documenting that, after adjustment for adult BMI, no association was observed for waist circumference, but an inverse one was observed for waist-to-hip ratio 15 . Similar to the findings of that review, we described sex-specific non-linear associations between birth weight and measurements of obesity/central obesity in women when assessed by BMI, waist circumference and wait-to-hip ratio, although only the latter was statistically significant in adjusted models 30 . These findings are consistent with another recent study among Chinese children and adolescents, which, while not presenting sex-specific analyses, also described J-shaped relationships between birth weight and waist-to-height ratios ${ }^{37}$. Likewise, low birth weight women of a younger cohort in Brazil showed higher rates of central obesity assessed by visceral fat 29. 


\section{Figure 3}

Odds of presenting a high (> 85 percentile) value for bioimpedance measures of body and truncal fat (kg), and \% body and truncal fat (fat/weight) according to birth weight. Brazilian Longitudinal Study of Adult Health (ELSA-Brasil) $(N=10,011)$.

3a) Body fat mass > p85 (men: $32 \mathrm{~kg}$; women: $37.9 \mathrm{~kg}$ )

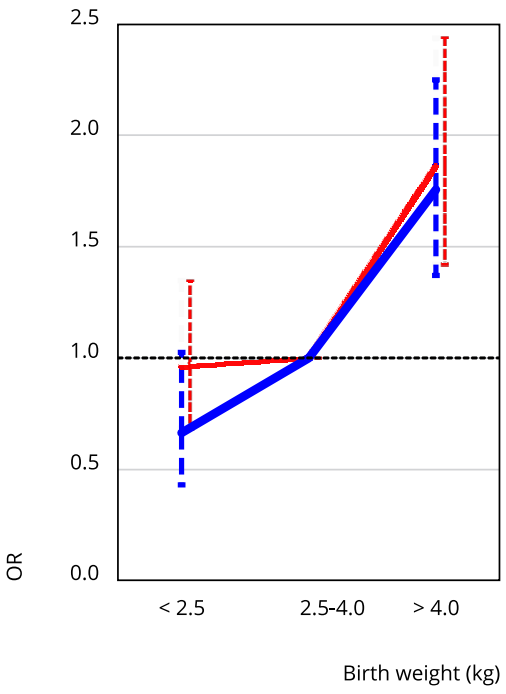

3c) Trucal body fat > p85 (men: $17.4 \mathrm{~kg}$; women: $19.5 \mathrm{~kg}$ )

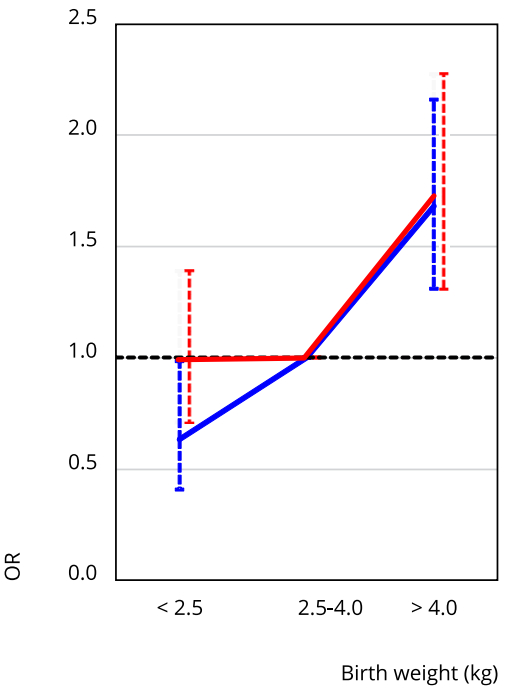

3b) \% body fat > p85 (men: $35.0 \%$; women: $46.3 \%$ )

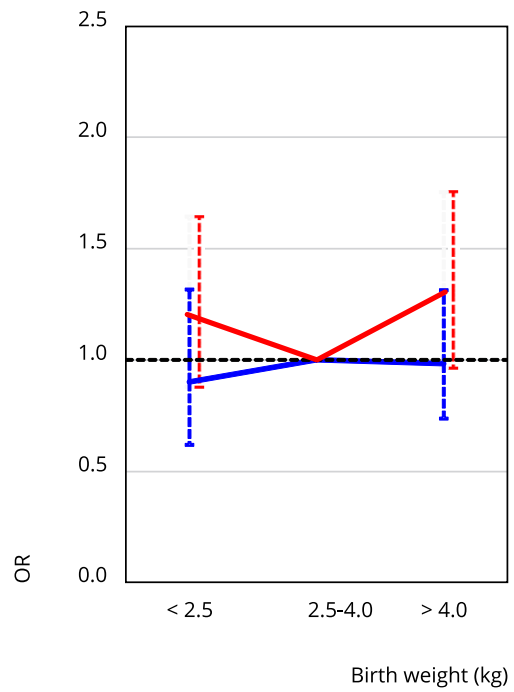

3d) \% truncal fat > p85 (men: $19.0 \%$; women: $23.8 \%$ )

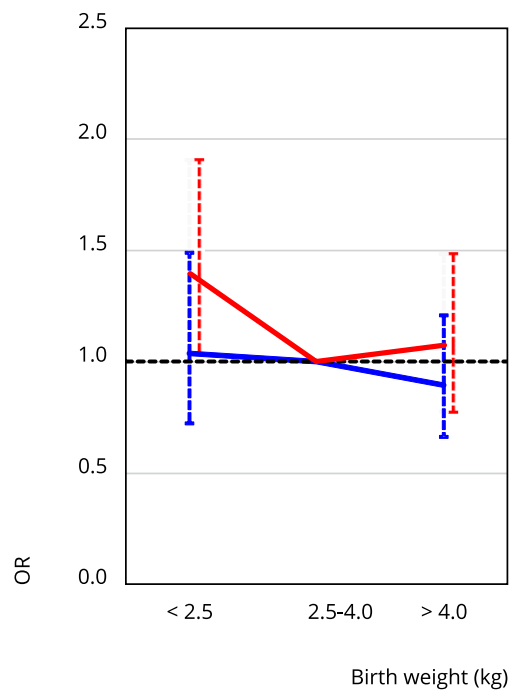

Notes: blue = men; red = women. Analyses were adjusted for study center, sex, age, family income, race/color, participant's and mother's educational attainment.

One should note that most studies describing linear associations between birth weight and anthropometric obesity indices in adults ${ }^{9}$ come from populations that are not suffering major fetal and early life undernutrition. Greater risk of chronic diseases associated with low birth weight is evident in settings of fetal and early life undernutrition, such as periods of famine 21,26,38, highlighting the need to avoid both low and high birth weight. According to Neel's hypothesis, "thrifty genes" selected during 
adversities across evolution may predispose to chronic diseases in modern times 39 . An alternative hypothesis, the thrifty phenotype hypothesis, was proposed later, relating intrauterine adversity with future chronic diseases 40,41,42. Based on studies of subjects born in periods of food restriction and war 21,26,38, intra-uterine growth retardation was proposed to cause future overall/central obesity. Interestingly, associations were observed among women but not among men 21,26, which is consistent with sex-specific mechanisms of intrauterine programming for future survival 16,18,20.

Mechanisms relating intrauterine environment, low and high birth weight, and future obesity/ central obesity include genetic 43 , epigenetic 9 , environmental 44,45 , and nutritional 46,47 factors. However, how these factors interact to explain the paradox of low and high birth weight causing future metabolic risk is still not clear ${ }^{48}$. It seems logical, though, to observe sex-specific patterns of associations such as those pointed out here, with women of low and high birth weight presenting greater proportions of body and truncal fat, as this may have explanations based on evolutionary necessities, with adaptations being present in utero to guarantee survival and reproduction. Throughout the life course, additional factors play a role, notably catch-up growth during childhood 49 and modern life styles which increase susceptibility for obesity, diabetes, and other chronic diseases.

A few potential limitations of our study merit discussion. First, birth weight was obtained by interviews with our adult subjects. Participants initially indicated their estimated birth weight within predefined categories and then provided their best recollection of the exact weight. Although we encouraged them to inquire among relatives and search for documents to confirm the informed birth weight, only $52 \%$ were able to provide an exact birth weight. Second, fat mass was obtained by bioimpedance, which assumes constant hydration of the fat-free mass, which may not hold in the presence of disease 50 . However, our standardized protocol of measurement in the morning after an 8-16 hour fast and after voiding exercises minimized this possibility. Additionally, bioimpedance does not measure body fat directly, representing only an estimate, and does not differentiate subcutaneous from visceral fat as many body imaging techniques do 51 .

On the other hand, our study has several strengths. We are unaware of prior studies investigating the relationship of birth weight and adult adiposity that have been able to measure body fat as we, have using bioimpedance. Our expressive sample size and multicentric design carried out in a free-living population expands on previous studies that have explored the subject, which were most conducted with individuals of European ancestry and in a historical and sociocultural situation distinct from that now experienced by most of the world's population, who live in low- and middle-income countries such as Brazil.

In conclusion, we found associations of high birth weight with larger total body and truncal fat mass, in men and women, and U-shaped associations between birth weight and relative total and truncal fat, most notably in women. These results add further and more detailed information to the associations previously described in ELSA-Brasil by anthropometric measures. They suggest that low and high birth weight contribute to adult central obesity, highlighting the need to prevent both when formulating nutritional policies during pregnancy. 


\section{Contributors}

All authors participated in analysis and interpretation of data, in writing the article, providing critical review of the intellectual content and approved the final version of the manuscript, and are responsible for all aspects of the work in ensuring its accuracy and integrity.

\section{Additional informations}

ORCID: Carolina Breda Resende (0000-0003-08529454); Vivian Cristine Luft (0000-0003-2371-9264); Bruce Duncan (0000-0002-7491-2630); Rosane Harter Griep (0000-0002-6250-2036); Maria del Carmen Bisi Molina (0000-0002-8614-988X); Sandhi Maria Barreto (0000-0001-7383-7811); Maria Inês Schmidt (0000-0002-3837-0731).

\section{Acknowledgments}

To Brazilian Ministry of Health, Brazilian Ministry of Science, Technology and Innovation, and Brazilian Graduate Studies Coodinating Board (CAPES) for the financial support. The ELSA-Brasil study was supported by the Brazilian Ministry of Health, Brazilian Ministry of Science, Technology and Innovation, and the Brazilian National Research Council (CNPq).

\section{References}

1. GBD 2016 Risk Factors Collaborators. Global, regional, and national comparative risk assessment of 84 behavioural, environmental and occupational, and metabolic risks or clusters of risks, 1990-2016: a systematic analysis for the Global Burden of Disease Study 2016. Lancet 2017; 390:1345-422.

2. NCD Risk Factor Collaboration. Trends in adult body-mass index in 200 countries from 1975 to 2014: a pooled analysis of 1698 population-based measurement studies with 19,2 million participants. Lancet 2016; 387:137796.

3. Haslam DW, James WPT. Obesity. Lancet 2005; 366:1197-209.

4. Tchernof A, Despres J-P. Pathophysiology of human visceral obesity: an update. Physiol Rev 2013; 93:359-404.

5. González-Muniesa P, Mártinez-González M-A, Hu FB, Després J-P, Matsuzawa Y, Looset JF, et al. Obesity. Nat Rev Dis Prim 2017; 3:17034.

6. Barker DJP, Eriksson JG, Forsén T, Osmond C. Fetal origins of adult disease: strength of effects and biological basis. Int J Epidemiol 2002; 31:1235-9.

7. Druet C, Ong KK. Early childhood predictors of adult body composition. Best Pract Res Clin Endocrinol Metab 2008; 22:489-502.

8. Calkins K, Devaskar SU. Fetal origins of adult disease. Curr Probl Pediatr Adolesc Health Care 2011; 41:158-76.

9. Schellong K, Schulz S, Harder T, Plagemann A. Birth weight and long-term overweight risk: systematic review and a meta-analysis including 643,902 persons from 66 studies and 26 countries globally. PLoS One 2012; 7:e47776.

10. Tomar AS, Tallapragada DSP, Nongmaithem SS, Shrestha S, Yajnik CS, Chandak GR. Intrauterine programming of diabetes and adiposity. Curr Obes Rep 2015; 4:418-28.

11. Saffery RNB. Epigenetics as the mediator of fetal programming of adult onset disease: what is the evidence? Acta Obstet Gynecol Scand 2014; 93:1090-8.

12. Qasim A, Turcotte M, Souza RJ, Samaan MC, Champredon D, Dushoff J, et al. On the origin of obesity: identifying the biological, environmental and cultural drivers of genetic risk among human populations. Obes Rev 2018; 19:121-49.

13. Lee SW, Son JY, Kim JM, Hwang SS, Han JS, Heo NJ. Body fat distribution is more predictive of all-cause mortality than overall adiposity. Diabetes Obes Metab 2018; 20:141-7.

14. Rogers I. The influence of birthweight and intrauterine environment on adiposity and fat distribution in later life. Int J Obes Relat Metab Disord 2003; 27:755-77. 
15. França GVA, Mola CL, Victora CG. Etiology and pathophysiology size at birth and abdominal adiposity in adults: a systematic review and meta-analysis. Obes Rev 2014; 15:77-91.

16. Trivers RL, Willard DE. Natural selection of parental ability to vary the sex ratio of offspring. Science 1973; 179:90-2.

17. Power ML, Schulkin J. Sex differences in fat storage, fat metabolism, and the health risks from obesity: possible evolutionary origins. $\mathrm{Br}$ J Nutr 2008; 99:931-40.

18. Sandman C, Glynn L, Davis E. Is there a viability-vulnerability tradeoff? Sex differences in fetal programming. J Psychosom Res 2013; 75:327-35.

19. White UA, Tchoukalova YD. Sex dimorphism and depot differences in adipose tissue function. Biochim Biophys Acta 2015; 1842:37792.

20. Dipietro JA, Voegtline KM. The gestational foundation of sex differences in development and vulnerability. Neuroscience 2017; 342:420.

21. Ravelli AC, Osmond C. Obesity at the age of 50 years in men and women exposed to famine prenatally. Am J Clin Nutr 1999; 70:811-6.

22. Luo Z, Mu R, Zhang X. Famine and overweight in China. Review of Agricultural Economics 2006; 28:296-304

23. Labayen I, Moreno LA, Ruiz JR, GonzálezGross M, Wärnberg J, Breidenassel C, et al. Small birth weight and later body composition and fat distribution in adolescents: the AVENA study. Obesity (Silver Spring) 2008; 16:1680-6.

24. Fung W. Early childhood malnutrition and adult obesity: evidence from the 1959-61 China famine. http://citeseerx.ist.psu.edu/view doc/download?doi=10.1.1.189.1517\&rep=rep $1 \&$ type $=$ pdf $($ accessed on 06/Jun/2020).

25. Yang T, Cairns B, Beral V. Association between birthweight and obesity in adult females. J Epidemiol Community Health 2010; 64 Suppl 1:A18-9.

26. Imai CM, Halldorsson TI, Gunnarsdottir I, Gudnason V, Aspelund T, Jonsson G, et al. Effect of birth year on birth weight and obesity in adulthood: comparison between subjects born prior to and during the great depression in Iceland. PLoS One 2012; 7:e44551.

27. Rønn PF, Smith LS, Andersen GS, Carstensen $\mathrm{B}$, Bjerregaard P, Jørgensen ME. Birth weight and risk of adiposity among adult Inuit in Greenland. PLoS One 2014; 9:e115976.

28. Tam C, Wang Y, Luan J, Lee H, Luk A, Tutino G. Non-linear relationship between birthweight and cardiometabolic risk factors in Chinese adolescents and adults. Diabet Med 2015; 32:220-5

29. França GVA, Rolfe EDL, Horta BL, Gigante DP, Yudkin JS, Ong KK, et al. Associations of birth weight, linear growth and relative weight gain throughout life with abdominal fat depots in adulthood: the 1982 Pelotas (Brazil) birth cohort study. Int J Obes (Lond) 2016; 40:1421.
30. Rockenbach G, Luft VC, Mueller NT, Duncan $\mathrm{BB}$, Stein MC, Vigo A, et al. Sex-specific associations of birth weight with measures of adiposity in mid-to-late adulthood: the Brazilian Longitudinal Study of Adult Health (ELSABrasil). Int J Obes (Lond) 2016; 40:1286-91.

31. Yarmolinsky J, Mueller NT, Duncan BB, Chor D, Bensenor IM, Griep RH, et al. Sexspecific associations of low birth weight with adult-onset diabetes and measures of glucose homeostasis: Brazilian Longitudinal Study of Adult Health. Sci Rep 2016; 6:37032.

32. Scafoglieri A, Clarys JP, Cattrysse E, Bautmans I. Use of anthropometry for the prediction of regional body tissue distribution in adults: benefits and limitations in clinical practice. Aging Dis 2014; 5:373-93.

33. Vecchié A, Dallegria F, Carbone F, Bonaventura A, Liberale L, Portincasa P, et al. Obesity phenotypes and their paradoxical association with cardiovascular diseases. Eur J Intern Med 2018; 48:6-17.

34. Schmidt MI, Duncan BB, Mill JG, Lotufo PA, Chor D, Barreto SM, et al. Cohort profile: Longitudinal Study of Adult Health (ELSABrasil). Int J Epidemiol 2015; 44:68-75.

35. Schmidt MI, Griep RH, Passos MV, Luft VC, Goulart AC, Menezes GMS, et al. Strategies and development of quality assurance and control in the ELSA-Brasil. Rev Saúde Pública 2013; 47 Suppl 2:105-12.

36. Desquilbet L, Mariotti F. Dose-response analyses using restricted cubic spline functions in public health research. Stat Med 2010; 29:1037-57.

37. Yuan ZP, Yang M, Liang L, Fu J-F, Xiong F, Liu G-L, et al. Possible role of birth weight on general and central obesity in Chinese children and adolescents: a cross-sectional study. Ann Epidemiol 2015; 10:748-52.

38. Ravelli GP, Stein ZA, Susser MW. Obesity in young men after exposure in utero and early infancy. N Engl J Med 1976; 295:349-53.

39. Neel JV. Diabetes mellitus: a "thrifty" genotype rendered detrimental by "progress"? Am J Hum Genet 1962; 14:353-62.

40. Hales CN, Barker DJP, Clark PMS, Cox LJ, Fall $\mathrm{C}$, Osmond $\mathrm{C}$, et al. Fetal and infant growth and impaired glucose tolerance at age $64 . \mathrm{Br}$ Med J 1991; 303:1019-22.

41. Barker DJ, Hales CN, Fall CH, Osmond C, Phipps K, Clark PM. Type 2 (non-insulin-dependent) diabetes mellitus, hypertension and hyperlipidaemia (syndrome X): relation to reduced fetal growth. Diabetologia 1993; 36:627.

42. Hale CN, Barker DJ. The thrifty phenotype hypothesis. Br Med Bull 2001; 60:5-20.

43. Goodarzi MO. Genetics of obesity: what genetic association studies have taught us about the biology of obesity and its complications. Lancet Diabetes Endocrinol 2018; 6:223-36. 
44. Sheikh AB, Nasrullah A, Haq S, Akhtar A, Ghazanfar H, Nasir A, et al. The interplay of genetics and environmental factors in the development of obesity. Cureus 2017; 9:e1435.

45. Barczyk A, Kutkowska-Kaźmierczak A, Castañeda J, Obersztyn E. The genetics of obesity - pathogenetic, clinical and diagnostic aspects. Dev Period Med 2017; 21:186-202.

46. Joffe YT, Houghton CA. A novel approach to the nutrigenetics and nutrigenomics of obesity and weight management. Curr Oncol Rep 2016; 18:43.

47. Castillo JJ, Orlando RA, Garver WS. Gene-nutrient interactions and susceptibility to human obesity. Genes Nutr 2017; 12:29.
48. Ornoy A. Prenatal origin of obesity and their complications: Gestational diabetes, maternal overweight and the paradoxical effects of fetal growth restriction and macrosomia. Reprod Toxicol 2011; 32:205-12.

49. Dulloo AG, Jacquet J, Seydoux J, Montani J-P. The thrifty 'catch-up fat' phenotype: its impact on insulin sensitivity during growth trajectories to obesity and metabolic syndrome. Int $\mathrm{J}$ Obes (Lond) 2006; 30 Suppl 4:S23-35.

50. Thibault R, Genton L, Pichard C. Body composition: why, when and for who? Clin Nutr 2012; 31:435-47.

51. Fosbol MO, Zerahn B. Contemporary methods of body composition measurement. Clin Physiol Funct Imaging 2015; 35:81-97. 


\section{Resumo}

A vida intrauterina é um período crítico para o desenvolvimento da gordura corporal e risco metabólico. O estudo investigou as associações entre peso ao nascer e gordura corporal total e de tronco em adultos. Analisamos os dados de 10.011 participantes do Estudo Longitudinal de Saúde no Adulto (ELSA-Brasil), com peso ao nascer de $<2,5 \mathrm{~kg}, 2,5-4,0 \mathrm{~kg}$ ou $>4,0 \mathrm{~kg}$, autorrelatado na linha de base (2008-2010) e que fizeram exame de bioimpedância na visita seguinte (2012-2014). A gordura corporal total e de tronco mais elevada estava associada com peso ao nascer elevado, quando comparado ao peso adequado $(p<0,001)$ em ambos os sexos (gordura total: 25,2 vs. $23,1 \mathrm{~kg}$ em homens e 31,4 vs. $27,7 \mathrm{~kg}$ em mulheres, e gordura de tronco: 13,5 vs. $12,4 \mathrm{~kg}$ em homens e 15,9 vs. $14,2 \mathrm{~kg}$ em mulheres). Foram observados padrões em " $U$ " nas análises de splines cúbicos restritos, no subconjunto de 5.212 individuos que informaram o peso ao nascer com exatidão, embora com significância estatística apenas para aqueles com peso ao nascer alto. Na amostra total, o peso ao nascer alto (comparado com o adequado) predizia (> percentil 85) gordura corporal total e de tronco, respectivamente: $O R=1,76$, IC95\%: 1,37-2,25 (homens) $e$ $O R=1,86$, IC95\%: 1,42-2,44 (mulheres); $O R=$ 1,68, IC95\%: 1,31-2,16 (homens) e OR $=1,73$, IC95\%: 1,31-2,28 (mulheres). Entretanto, baixo peso ao nascer predizia gordura de tronco elevada (> percentil 85) apenas nas mulheres $(\mathrm{OR}=1,40$, IC95\%: 1,03-1,91). O estudo conclui que nesse grupo de homens e mulheres que nasceram numa época em que a desnutrição fetal era prevalente, $o$ peso ao nascer mostrou associações complexas, frequentemente não lineares, com a gordura corporal na idade adulta, o que enfatiza a necessidade de intervenções para prevenir, durante a gestação, $o$ baixo e alto peso ao nascer.

Obesidade; Peso ao Nascer; Composição Corporal; Tecido Adiposo; Estudo Observacional

\section{Resumen}

La vida intrauterina es un periodo crítico para el desarrollo de la masa de grasa corporal y riesgo metabólico. Investigamos las asociaciones entre peso al nacer y la grasa total y troncal en adultos. Analizamos datos de 10.011 adultos que participaron en el Estudio Longitudinal de Salud en Adultos (ELSA-Brasil) quienes autoinformaron de un peso al nacer $<2,5 \mathrm{~kg}, 2,5-4,0 \mathrm{~kg}, o>4,0 \mathrm{~kg}$ en la base de referencia (2008-2010) y experimentaron bioimpedancia en la siguiente visita de seguimiento (2012-2014). La mayor media de masa grasa total y troncal se observó en quienes tuvieron un alto peso al nacer, en comparación con quienes tuvieron un adecuado peso al nacer $(p<0,001)$ en ambos sexos (grasa total: $25,2 \mathrm{vs}$. $23,1 \mathrm{~kg}$ en hombres y $31,4 \mathrm{vs}$. $27,7 \mathrm{~kg}$ en mujeres, $y$ grasa troncal: 13,5 vs. $12,4 \mathrm{~kg}$ en hombres y 15,9 vs. $14,2 \mathrm{~kg}$ en mujeres). Se observaron patrones en forma de " $U$ " en análisis spline cúbicos restringidos en el subconjunto de 5.212 personas que informaron de sus pesos exactos al nacer, pese a que eran estadísticamente significativos solamente quienes tenían un alto peso al nacer. En toda la muestra con alto peso al nacer, comparada con el adecuado peso al nacer, se pronosticó contar con más masa grasa total $y$ troncal (> percentil 85), respectivamente: $O R=$ 1,76, IC95\%: 1,37-2, 25 (hombres) y $\mathrm{OR}=1,86$, IC95\%: 1,42-2, 44 (mujeres); OR = 1,68, IC95\%: 1,31-2,16 (hombres) y $\mathrm{OR}=1,73$, IC 95\%: 1,312,28 (mujeres). No obstante, contar con un bajo peso al nacer predispuso a contar con más masa grasa troncal solamente en mujeres (> percentil 85) $\%(O R=1,40$, IC95\%: 1,03-1,91). En conclusión, en estos hombres y mujeres nacidos durante un período en el que la malnutrición fetal era prevalente, el peso al nacer mostró frecuentemente asociaciones no lineales complejas, con grasa corporal en la etapa adulta, resaltando la necesidad de intervenciones para prevenir el bajo y el alto peso al nacer durante el embarazo.

Obesidad; Peso al Nacer; Composición Corporal; Tejido Adiposo; Estudio Observacional
Submitted on $17 / \mathrm{Feb} / 2020$

Final version resubmiettd on 22/Jun/2020

Approved on 06/Jul/2020 